\title{
Blood alkaline phosphatase predicts prognosis of patients with advanced HER2-negative gastric cancer receiving immunotherapy
}

\author{
Jianli Hu, Shengli Yang, Jing Wang, Qiuyue Zhang, Lei Zhao, Dejun Zhang, Dandan Yu, Min Jin, \\ Hong Ma, Hongli Liu, Jun Xue, Tao Zhang
}

Cancer Center, Union Hospital, Tongii Medical College, Huazhong University of Science and Technology, Wuhan, China

Contributions: (I) Conception and design: J Hu, S Yang, J Wang, T Zhang; (II) Administrative support: H Liu, T Zhang; (III) Provision of study materials or patients: Q Zhang, L Zhao, D Zhang, J Xue; (IV) Collection and assembly of data: D Yu, M Jin, H Ma, H Liu; (V) Data analysis and interpretation: J Hu, S Yang; (VI) Manuscript writing: All authors; (VII) Final approval of manuscript: All authors.

Correspondence to: Tao Zhang. Cancer Center, Union Hospital, Tongji Medical College, Huazhong University of Science and Technology, 1277 Jiefang Avenue, Wuhan 430022, China. Email: taozhangxh@hust.edu.cn.

Background: Immune checkpoint blockade is effective against many cancer types, but few patients achieve a complete response (OR). Therefore, effective prognostic biomarkers are needed for metastatic gastric cancer (GC) patients after immune treatment. The present study assessed the value of hematological parameters as markers of the effectiveness of immune checkpoint blockade among metastatic GC patients.

Methods: This retrospective study included patients with metastatic GC who underwent multiline chemotherapy including at least two courses of immunotherapy between September 2018 and December 2020. Patient and tumor characteristics were tested for prognostic significance by analysis of variance or chisquare test. Kaplan-Meier and Cox analyses were performed to identify factors associated with progressionfree survival (PFS).

Results: Sixty-one GC patients (mean age 55.61 \pm 11.97 years, range 23-80 years, 24 females, and 37 males) were included, and 27, 9 and 25 cases had organ only, peritoneum only, and simultaneous organ and peritoneum metastasis, respectively. Gastrectomy was performed in 24 cases, and there was no operative treatment in the other 37 cases, while all patients received two or more lines of chemotherapy. After immune treatment, 13 patients achieved a partial response (PR), 16 stable disease (SD), and 32 progressive disease (PD). The median PFS was 4.93 \pm 3.47 months. An alkaline phosphatase (ALP) level $>225 \mathrm{U} / \mathrm{L}$, a lactate dehydrogenase level $(\mathrm{LDH})>299 \mathrm{U} / \mathrm{L}$, and a body mass index $(\mathrm{BMI})>24 \mathrm{~kg} / \mathrm{m}^{2}$ were associated with a short PFS ( $\mathrm{P}=0.01, \mathrm{P}=0.008$, and $\mathrm{P}=0.039$, respectively). A Cox multivariate proportional hazard model indicated that higher ALP level was a significant prognostic indicator for adverse PFS.

Conclusions: Our data show an ALP cutoff of 225 U/L offered good prognostic sensitivity for HER2negative metastatic GC. ALP measurement represents a convenient, cost-effective, and relatively sensitive screening tool, and prospective studies involving its evaluation in addition to other biomarkers in metastatic GC patients are indicated.

Keywords: Gastric cancer (GC); prognosis; progression-free survival (PFS); immune treatment

Submitted Jun 10, 2021. Accepted for publication Aug 11, 2021.

doi: $10.21037 /$ atm-21-3376

View this article at: https://dx.doi.org/10.21037/atm-21-3376

\section{Introduction}

China has one of the highest incidence rates of gastric cancer (GC) worldwide, and GC is the second most common cause of cancer-related death in our country
$(1,2)$. While surgical treatment is the only curative therapy for GC, it can only be performed in selected patients in the early stages of the disease, and treatment for advanced GC usually includes a combination of 
chemotherapy, radiotherapy, molecular-targeted therapy, and immunotherapy (3). Unfortunately, many patients develop local or distant metastasis after gastrectomy, and for recurrent or metastatic gastric cancer (mGC), firstline chemotherapy is based on platinum, fluoropyrimidine, and trastuzumab if the tumor is human epidermal growth factor 2 (HER2)-positive (4). Second-line drugs include docetaxel, irinotecan, and the vascular endothelial growth factor monoclonal antibody, which have a limited effect on the PFS and overall survival (OS) of patients (5). However, the role of systemic chemotherapy in HER2negative GC patients is limited. Current clinical guidelines do not recommend a standard maintenance chemotherapy for advanced or metastatic HER2-negative GC, because most reported clinical trials could not reach their primary endpoints.

In recent years, immune checkpoint inhibitors (ICIs), represented by programmed death-1 (PD-1) receptor or ligand (PD-L1) inhibitors, have changed the treatment approach for various cancers $(6,7)$. ICIs may be effective in GC because of a relatively high mutational load (8), and clinical trials with pembrolizumab or nivolumab showed a wide range of response (10-26\%) in mGC in the salvage setting (9-11). Currently, pembrolizumab and nivolumab have been approved for the treatment of recurrent locally advanced GC or mGC with PD-L1 positivity in the United States and Japan, respectively $(12,13)$.

However, ICIs are not effective in every patient with a particular type of cancer, and it is important to identify novel biological markers for predicting the response to their use $(14,15)$. Significant associations have been reported between high tumor mutational burden and response to ICIs in a variety of tumors (16). Additionally, studies have been conducted to identify routinely available blood and clinical markers such as white blood cell count, neutrophil count, platelet count, and lactate dehydrogenase (LDH) level, which can be used to predict the response and toxicity to ICIs (17). While many of these parameters have been found to be associated with poor outcomes in various cancers $(18,19)$, their ability to predict outcomes of patients with mGC receiving ICIs has not been reported. Compared with previous study, the present study was conducted to identify potential prognostic biomarkers for mGC patients after immunotherapy, which could help in planning individualized treatments for these patients. We present the following article in accordance with the REMARK reporting checklist (available at https://dx.doi.org/10.21037/ atm-21-3376).

\section{Methods}

\section{Patient selection and data collection}

This study was conducted at the Cancer Center, Union Hospital, Tongji Medical College, Huazhong University of Science and Technology, China. This was a retrospective analysis of the clinicopathological data of patients with metastatic/recurrent GC who received at least second-line chemotherapy between September 2018 and December 2020 and experienced tumor progression. All included patients received at least one ICI including nivolumab, pembrolizumab, toripalimab, sintilimab, tislelizumab, or camrelizumab, and received immunotherapy regimens intravenously every 3 weeks. The study was conducted in accordance with the Declaration of Helsinki (as revised in 2013). All patients or their legal representatives gave written informed consent for the treatment and inquiries related to this study, which was approved by the Ethics Committee of Tongji Medical College, Huazhong University of Science and Technology (No.:IORG0003571).

The inclusion criteria were: (I) age more than 18 years; (II) pathologically confirmed gastric adenocarcinoma; (III) Eastern Cooperative Oncology Group (ECOG) performance status of 1 or less; (IV) measurable disease as defined by Response Evaluation Criteria in Solid Tumors 1.1 (RECIST 1.1); and (V) good hepatic and renal function with normal hematological parameters. Patients with microsatellite instability high/deficient mismatch repair (MSI-H/dMMR), a history of autoimmune disorders, human immunodeficiency virus (HIV) infection, or hepatitis $\mathrm{B}$ or $\mathrm{C}$ virus infection were excluded.

Clinicopathological data and hematological parameters were retrieved from the medical records of patients before immunotherapy commenced. The staging of GC was according to the $8^{\text {th }}$ edition of the American Joint Committee on Cancer (AJCC) TNM staging system.

\section{Follow-up evaluation}

Patients were diagnosed and evaluated during follow-up by contrast enhanced computed tomography (CT) or magnetic resonance imaging (MRI) of the abdomen. The response to treatment was classified according to RECIST 1.1 criteria as complete response (OR), partial response (PR), stable disease (SD), or progressive disease (PD). Tumor response was evaluated every 6 weeks during the first 3 months and every 8 weeks thereafter using imaging studies. Adverse events were recorded as per the Common Terminology 
Table 1 Demographic and clinicopathological characteristics of the included patients

\begin{tabular}{|c|c|}
\hline Variable & $\mathrm{N}=61(\%)$ \\
\hline Median age, years [range] & $55.61 \pm 11.97[23-80]$ \\
\hline \multicolumn{2}{|l|}{ Sex } \\
\hline Male & $37(60.7)$ \\
\hline Female & $24(39.3)$ \\
\hline \multicolumn{2}{|l|}{ Race } \\
\hline Asian & $61(100.0)$ \\
\hline \multicolumn{2}{|l|}{$\mathrm{BMI}, \mathrm{kg} / \mathrm{m}^{2}$} \\
\hline$\leq 18$ & $7(11.5)$ \\
\hline $18-24$ & $45(73.8)$ \\
\hline$>24$ & $9(14.7)$ \\
\hline \multicolumn{2}{|l|}{ Line of immunotherapy } \\
\hline First-line & $21(34.4)$ \\
\hline Second-line & $21(34.3)$ \\
\hline Third-line or more & $19(31.2)$ \\
\hline \multicolumn{2}{|l|}{ Number of metastatic sites } \\
\hline 1 & $22(36.1)$ \\
\hline$\geq 2$ & $39(63.9)$ \\
\hline \multicolumn{2}{|l|}{ Previous gastrectomy } \\
\hline Yes & $24(39.3)$ \\
\hline No & $37(60.7)$ \\
\hline \multicolumn{2}{|c|}{ Combined with chemotherapy or targeted treatment } \\
\hline Yes & $45(73.8)$ \\
\hline No & $16(26.2)$ \\
\hline
\end{tabular}

Criteria for Adverse Events (CTCAE) system version 5.

\section{Definitions}

Progression-free survival (PFS) was defined as the time from the beginning of immunotherapy until the appearance of recurrence or death due to any cause. The last follow-up time was on February 20th, 2021. The objective response rate (ORR) included cases of CR and PR, and the disease control rate (DCR) considered the ORR plus cases of SD.

\section{Statistical analysis}

All statistical analyses were conducted using SPSS version 20.
Continuous variables are presented as mean \pm standard deviation. One-way analysis of variance (ANOVA) was used to identify significant differences between continuous variables, and categorical variables were compared by the chi-squared test. Kaplan-Meier survival curves were used to estimate PFS and OS, and univariate Cox regression analysis was used to determine the clinicopathological variables associated with survival. A $\mathrm{P}$ value $<0.05$ was considered statistically significant.

\section{Results}

\section{Patient characteristics}

A total of 61 patients were included in the study, and their main demographic, biochemical, and clinical details are presented in Table 1. There were 24 females and 37 males, and most $(63.9 \%)$ patients had more than one site of metastatic involvement. All patients were HER2 negative, and all received two or more lines of chemotherapy. The numbers of patients receiving one line, two lines, and three or more lines of chemotherapy was 21,21 , and 19 , respectively. Taxane-based chemotherapy was administered to 23 patients, nine received 5-FU-based chemotherapy, two received irinotecan-based chemotherapy, and five patients received a combination of antiangiogenic drugs.

\section{Clinical response and toxicity}

After immunotherapy, PR, SD, and PD were observed in 13 (21.3\%), 16 (26.2\%) and 32 (52.5\%) patients, respectively. The ORR was $21.3 \%$, DCR was $47.5 \%$, and the median PFS was $4.93 \pm 3.46$ months. Most patients experienced grade 1 or 2 side effects based on the CTCAE 5.0 criteria, and the most common were fatigue, headache, arthralgia, rash, pruritus, pneumonitis, diarrhea and/or colitis, hepatitis, and endocrinopathies. No patients developed grade $\geq 3$ hepatitis.

\section{Relationships between clinical parameters and survival outcomes}

The analysis showed that patients with elevated ALP levels (ALP >225 g/L) and increased LDH levels (LDH >299 U/L) were more likely to have a short PFS compared with those with lower levels $(\mathrm{P}=0.017$ and $\mathrm{P}=0.013$, respectively; Figure 1). Similarly, a higher BMI (BMI $\left.>24 \mathrm{~kg} / \mathrm{m}^{2}\right)$ was also associated with a short PFS $(\mathrm{P}=0.014$; Figure 1$)$. The albumin/globulin (A/G) level, A/L, and $\mathrm{r}$ glutamyl 

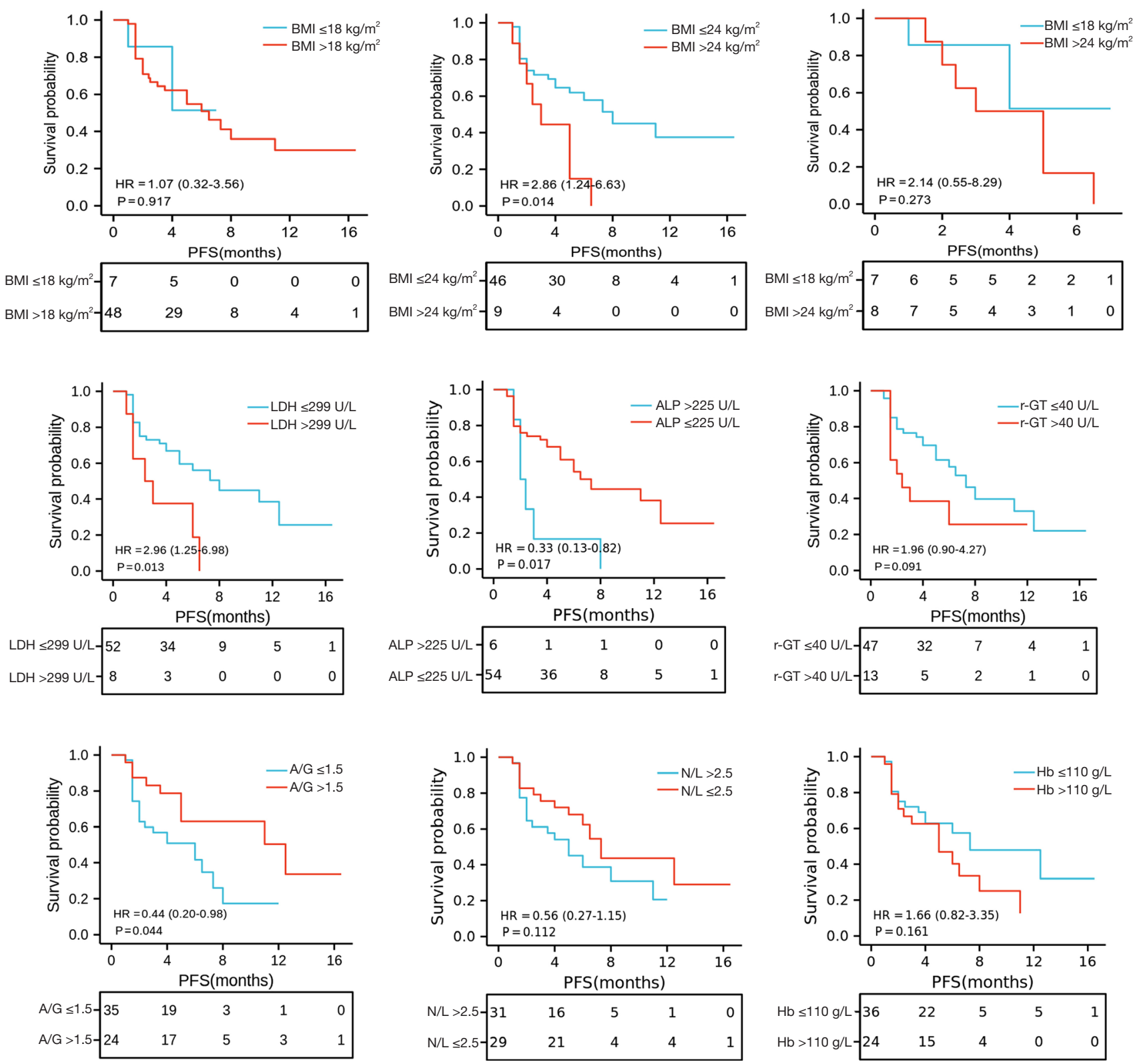

Figure 1 Cumulative progression-free survival of the included patients grouped according to various laboratory parameters. N/L, neutrophils/lymphocytes; r-GT, r glutamyl transpeptidase; ALP, alkaline phosphatase; LDH, lactate dehydrogenase; A/G, albumin/globulin; BMI, body mass index.

transpeptidase (r-GT) level individually had no significant impact on PFS (Figure 1).

\section{Prognostic factors for PFS}

Univariate analysis showed ALP level $(\mathrm{P}=0.019)$, LDH level $(\mathrm{P}=0.016)$, and $\mathrm{BMI}$ were risk factors for PFS, and the multivariate Cox proportional hazards model identified
ALP $[\mathrm{P}=0.023$, hazard ratio $(\mathrm{HR})=2.901,95 \%$ confidence interval (CI): 1.158-7.269] as an independent risk factor for PFS (Table 2).

\section{Discussion}

Early-phase clinical trials have demonstrated the potential benefits of PD-1 therapy in patients with MSI-H or positive 
Table 2 Univariate and multivariate analyses of factors associated with PFS

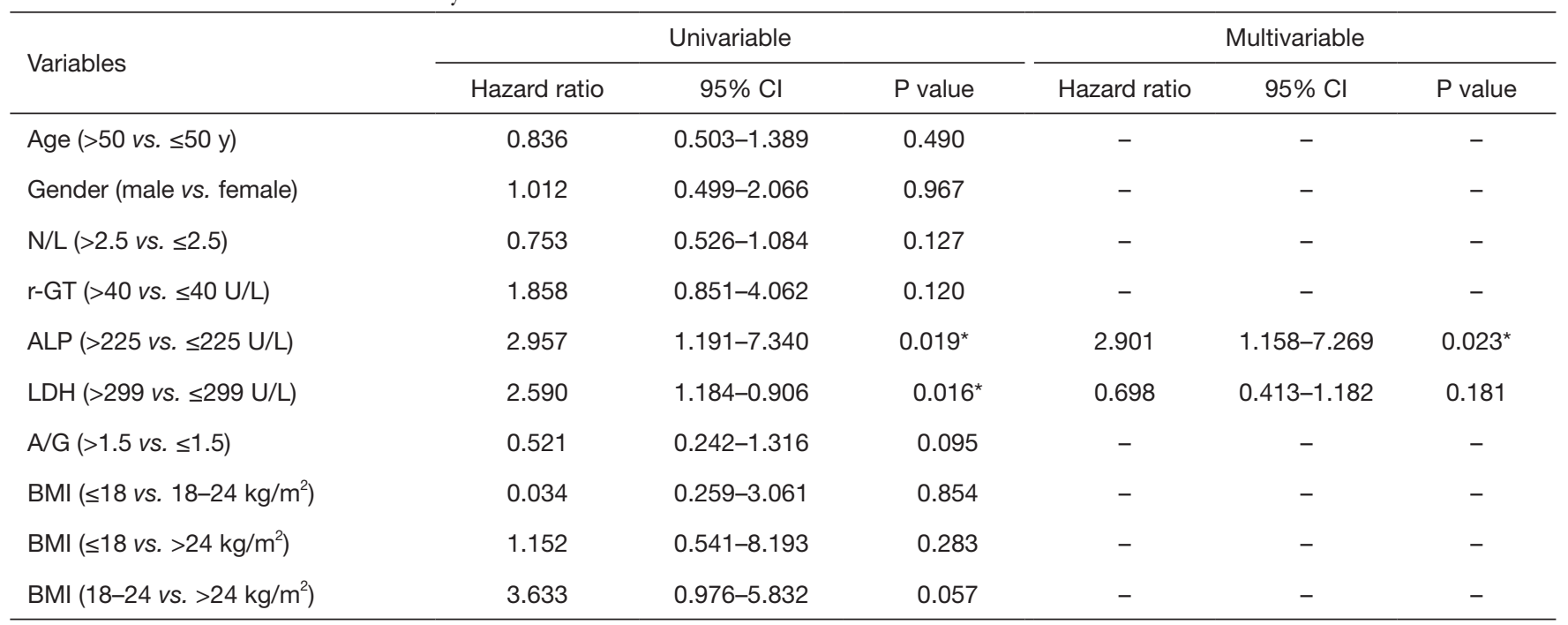

*, P<0.05. N/L, neutrophils/lymphocytes; r-GT, $r$ glutamyl transpeptidase; ALP, alkaline phosphatase; LDH, lactate dehydrogenase; A/G, albumin/globulin; BMI, body mass index.

PD-L1 advanced GC (20). In the present study, we found that nearly half of the patients with HER2-negative locally advanced, metastatic, or recurrent GC had PD or SD upon treatment with different PD-1 inhibitors, and all patients achieved nearly 5 months of PFS. These findings suggest that some patients with HER2-negative GC can benefit from PD-1 inhibitors in clinical practice. However, future studies with a longer follow-up are required to identify the potential benefits of ICIs in improving OS.

Several groups have explored the role of clinicopathological factors in predicting GC prognosis, but these factors have been of limited value in clinical practice (21-24). Recently, blood biomarkers have become attractive and convenient prognostic markers for GC (25), and many researchers have focused on using these indicators to determine prognosis based on routine blood markers after gastrectomy $(26,27)$. In the present study, we analyzed the role of various clinical characteristics and routine blood parameters in predicting the response to immunotherapy in terms of PFS. Several clinical studies have reported the neutrophil-to-lymphocyte ratio $(\mathrm{N} / \mathrm{L})$, platelet-to-lymphocyte ratio (P/L), and monocyteto-lymphocyte ratio $(\mathrm{M} / \mathrm{L})$ to be inversely correlated with the prognosis of GC $(28,29)$. Nevertheless, the role of these biochemical parameters in predicting the response of ICIs in GC is not clear. ALP is a vital component of liver function tests and is elevated in patients with liver disease or bile duct obstruction. Recently, ALP has been found to be an independent prognostic biomarker in some types of cancers (30). In addition, LDH is another useful prognostic factor in cancer patients (24). LDH is generated during the glycolytic activity of the cells, and is greatly enhanced in tumor cells, especially in the presence of hypoxia and a high tumor burden. Clinical studies have reported that melanoma patients with elevated LDH levels have a poorer response to immunotherapy than those with normal $\mathrm{LDH}$ levels (30). In the present study, we found that higher ALP and LDH levels were associated with shorter PFS following immunotherapy in GC.

Obesity is a risk factor for the development of many acute and chronic diseases including cancers (31-33). Moreover, studies have found that an elevated BMI is associated with reduced cancer survival (34). These observations are likely related to elevated levels of chronic inflammation and high adiposity (35-37). Contrastingly, some recent studies found that BMI is associated with improved survival in colorectal cancer $(38,39)$. Due to the lack of consensus, we aimed to explore the impact of BMI on the survival of GC patients and their response to ICIs. While our data suggest a probable survival benefit of ICIs in GC patients with a lower BMI, due to the small sample size, future studies are required to establish the relationship between $\mathrm{BMI}$ and $\mathrm{PFS}$ in patients with HER2-negative mGC.

In addition, Cox multivariate analysis identified ALP as an independent predictor of PFS, although we cannot explain the reason behind this association. As it has been 


\section{Page 6 of 8}

reported that chronic liver disease and bone metastasis can increase ALP levels, future studies are needed to understand the underlying mechanisms responsible for the association between ALP and prognosis of GC.

There are some limitations to the present study. First, the study was conducted in a single center with a small sample size, and was fundamentally retrospective. Second, the criteria used to include patients were very restrictive, as those with MSI-H/dMMR, incomplete medical records, or lack of follow-up data were excluded. Third, all possible factors may affect the PFS were not studied. Hence, future prospective, multicenter studies with a larger sample size are needed to improve the quality of evidence derived from the present study.

In conclusion, our data show that an ALP level $>225 \mathrm{U} / \mathrm{L}$ was associated with a poor response to ICIs and a decreased PFS in patients with HER2-negative mGC. ALP can be used as a convenient, cost-effective and relatively sensitive screening tool for the selection of HER2-negative mGC patients for ICIs. Prospective studies involving ALP add with other biomarkers are required to determine their actual prognostic significance in mGC.

\section{Acknowledgments}

Funding: This study was supported by the National Key R\&D Program of China (2018YFC1313300), the Natural Science Foundation of China (Grant No. 81874061), and the Huazhong University of Science and Technology Undergraduate Innovation and Entrepreneurship Training Program (DYLC2021072).

\section{Footnote}

Reporting Checklist: The authors have completed the STROBE reporting checklist. Available at https://dx.doi. org/10.21037/atm-21-3376

Data Sharing Statement: Available at https://dx.doi. org/10.21037/atm-21-3376

Conflicts of Interest: All authors have completed the ICMJE uniform disclosure form (available at https://dx.doi. org/10.21037/atm-21-3376). The authors have no conflicts of interest to declare.

Ethical Statement: The authors are accountable for all aspects of the work in ensuring that questions related

\section{Hu et al. ALP as a prognostic marker in metastatic gastric cancer}

to the accuracy or integrity of any part of the work are appropriately investigated and resolved. The study was conducted in accordance with the Declaration of Helsinki (as revised in 2013). The study was approved by the Ethics Committee of Tongji Medical College, Huazhong University of Science and Technology (No.: IORG0003571). All patients or their legal representatives gave written informed consent for the treatment and inquiries related to this study.

Open Access Statement: This is an Open Access article distributed in accordance with the Creative Commons Attribution-NonCommercial-NoDerivs 4.0 International License (CC BY-NC-ND 4.0), which permits the noncommercial replication and distribution of the article with the strict proviso that no changes or edits are made and the original work is properly cited (including links to both the formal publication through the relevant DOI and the license). See: https://creativecommons.org/licenses/by-nc-nd/4.0/.

\section{References}

1. Smyth EC. Chemotherapy for resectable microsatellite instability-high gastric cancer? Lancet Oncol 2020;21:204.

2. Eusebi LH, Telese A, Marasco G, et al. Gastric cancer prevention strategies: A global perspective. J Gastroenterol Hepatol 2020;35:1495-502.

3. Song Z, Wu Y, Yang J, et al. Progress in the treatment of advanced gastric cancer. Tumour Biol 2017;39:1010428317714626.

4. Parisi A, Porzio G, Ficorella C. Multimodality Treatment in Metastatic Gastric Cancer: From Past to Next Future. Cancers (Basel) 2020;12:2598.

5. Ghosn M, Tabchi S, Kourie HR, et al. Metastatic gastric cancer treatment: Second line and beyond. World J Gastroenterol 2016;22:3069-77.

6. Johnson DB, Sullivan RJ, Menzies AM. Immune checkpoint inhibitors in challenging populations. Cancer 2017;123:1904-11.

7. Gobbini E, Charles J, Toffart AC, et al. Current opinions in immune checkpoint inhibitors rechallenge in solid cancers. Crit Rev Oncol Hematol 2019;144:102816.

8. Kim ST, Cristescu R, Bass AJ, et al. Comprehensive molecular characterization of clinical responses to PD-1 inhibition in metastatic gastric cancer. Nat Med 2018;24:1449-58.

9. Kang YK, Boku N, Satoh T, et al. Nivolumab in patients with advanced gastric or gastro-oesophageal 
junction cancer refractory to, or intolerant of, at least two previous chemotherapy regimens (ONO-4538-12, ATTRACTION-2): a randomised, double-blind, placebocontrolled, phase 3 trial. Lancet 2017;390:2461-71.

10. Muro K, Chung HC, Shankaran V, et al. Pembrolizumab for patients with PD-L1-positive advanced gastric cancer (KEYNOTE-012): a multicentre, open-label, phase 1b trial. Lancet Oncol 2016;17:717-26.

11. Bang YJ, Kang YK, Catenacci DV, et al. Pembrolizumab alone or in combination with chemotherapy as firstline therapy for patients with advanced gastric or gastroesophageal junction adenocarcinoma: results from the phase II nonrandomized KEYNOTE-059 study. Gastric Cancer 2019;22:828-37.

12. Fashoyin-Aje L, Donoghue M, Chen H, et al. FDA Approval Summary: Pembrolizumab for Recurrent Locally Advanced or Metastatic Gastric or Gastroesophageal Junction Adenocarcinoma Expressing PD-L1. Oncologist 2019;24:103-9.

13. Smyth E, Thuss-Patience PC. Immune Checkpoint Inhibition in Gastro-Oesophageal Cancer. Oncol Res Treat 2018;41:272-80.

14. Topalian SL, Taube JM, Anders RA, et al. Mechanismdriven biomarkers to guide immune checkpoint blockade in cancer therapy. Nat Rev Cancer 2016;16:275-87.

15. Meng X, Huang Z, Teng F, et al. Predictive biomarkers in PD-1/PD-L1 checkpoint blockade immunotherapy. Cancer Treat Rev 2015;41:868-76.

16. Hellmann MD, Callahan MK, Awad MM, et al. Tumor Mutational Burden and Efficacy of Nivolumab Monotherapy and in Combination with Ipilimumab in Small-Cell Lung Cancer. Cancer Cell 2018;33:853-861.e4.

17. Hopkins AM, Rowland A, Kichenadasse G, et al. Predicting response and toxicity to immune checkpoint inhibitors using routinely available blood and clinical markers. Br J Cancer 2017;117:913-20.

18. Mezquita L, Auclin E, Ferrara R, et al. Association of the Lung Immune Prognostic Index With Immune Checkpoint Inhibitor Outcomes in Patients With Advanced Non-Small Cell Lung Cancer. JAMA Oncol 2018;4:351-7.

19. Liu D, Wang D, Wu C, et al. Prognostic significance of serum lactate dehydrogenase in patients with breast cancer: a meta-analysis. Cancer Manag Res 2019;11:3611-9.

20. Vanderwalde A, Spetzler D, Xiao N, et al. Microsatellite instability status determined by next-generation sequencing and compared with PD-L1 and tumor mutational burden in 11,348 patients. Cancer Med 2018;7:746-56.
21. Chen YC, Fang WL, Wang RF, et al. Clinicopathological Variation of Lauren Classification in Gastric Cancer. Pathol Oncol Res 2016;22:197-202.

22. Han J, Tu J, Tang C, et al. Clinicopathological Characteristics and Prognosis of cT1N0M1 Gastric Cancer: A Population-Based Study. Dis Markers 2019;2019:5902091.

23. Lei YY, Huang JY, Zhao QR, et al. The clinicopathological parameters and prognostic significance of HER2 expression in gastric cancer patients: a meta-analysis of literature. World J Surg Oncol 2017;15:68.

24. Hu K, Wang S, Wang Z, et al. Clinicopathological risk factors for gastric cancer: a retrospective cohort study in China. BMJ Open 2019;9:e030639.

25. Huang XZ, Yang YC, Chen Y, et al. Preoperative Anemia or Low Hemoglobin Predicts Poor Prognosis in Gastric Cancer Patients: A Meta-Analysis. Dis Markers 2019;2019:7606128.

26. Abbas M, Habib M, Naveed M, et al. The relevance of gastric cancer biomarkers in prognosis and pre- and postchemotherapy in clinical practice. Biomed Pharmacother 2017;95:1082-90.

27. Matsuoka T, Yashiro M. Biomarkers of gastric cancer: Current topics and future perspective. World J Gastroenterol 2018;24:2818-32.

28. Sun X, Liu X, Liu J, et al. Preoperative neutrophil-tolymphocyte ratio plus platelet-to-lymphocyte ratio in predicting survival for patients with stage I-II gastric cancer. Chin J Cancer 2016;35:57.

29. Kim EY, Lee JW, Yoo HM, et al. The Platelet-toLymphocyte Ratio Versus Neutrophil-to-Lymphocyte Ratio: Which is Better as a Prognostic Factor in Gastric Cancer? Ann Surg Oncol 2015;22:4363-70.

30. Saif MW, Alexander D, Wicox CM. Serum Alkaline Phosphatase Level as a Prognostic Tool in Colorectal Cancer: A Study of 105 patients. J Appl Res 2005;5:88-95.

31. Karczewski J, Begier-Krasińska B, Staszewski R, et al. Obesity and the Risk of Gastrointestinal Cancers. Dig Dis Sci 2019;64:2740-9.

32. Avgerinos KI, Spyrou N, Mantzoros CS, et al. Obesity and cancer risk: Emerging biological mechanisms and perspectives. Metabolism 2019;92:121-35.

33. Iyengar NM, Gucalp A, Dannenberg AJ, et al. Obesity and Cancer Mechanisms: Tumor Microenvironment and Inflammation. J Clin Oncol 2016;34:4270-6.

34. Van Wilpe S, Koornstra R, Den Brok M, et al. Lactate dehydrogenase: a marker of diminished antitumor immunity. Oncoimmunology 2020;9:1731942. 


\section{Page 8 of 8}

35. Dev R, Bruera E, Dalal S. Insulin resistance and body composition in cancer patients. Ann Oncol 2018;29:ii18ii26.

36. Zimta AA, Tigu AB, Muntean M, et al. Molecular Links between Central Obesity and Breast Cancer. Int J Mol Sci 2019;20:5364.

37. Rose DP, Vona-Davis L. Biochemical and molecular mechanisms for the association between obesity, chronic inflammation, and breast cancer. Biofactors 2014;40:1-12.

38. Schlesinger S, Siegert S, Koch M, et al. Postdiagnosis

Cite this article as: $\mathrm{Hu} \mathrm{J}$, Yang S, Wang J, Zhang Q, Zhao L, Zhang D, Yu D, Jin M, Ma H, Liu H, Xue J, Zhang T. Blood alkaline phosphatase predicts prognosis of patients with advanced HER2-negative gastric cancer receiving immunotherapy. Ann Transl Med 2021;9(16):1316. doi: 10.21037/atm-21-3376

\section{Hu et al. ALP as a prognostic marker in metastatic gastric cancer}

body mass index and risk of mortality in colorectal cancer survivors: a prospective study and meta-analysis. Cancer Causes Control 2014;25:1407-18.

39. Amptoulach S, Gross G, Kalaitzakis E. Differential impact of obesity and diabetes mellitus on survival after liver resection for colorectal cancer metastases. J Surg Res 2015;199:378-85.

(English Language Editor: B. Draper) 